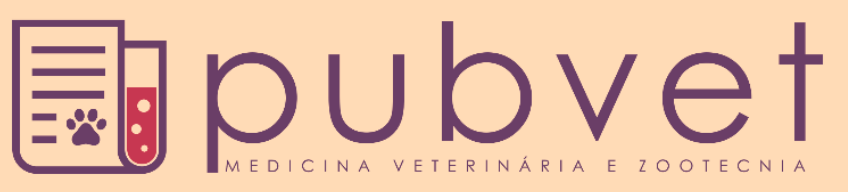

https://doi.org/10.31533/pubvet.v15n04a798.1-5

\title{
Sinais radiográficos de corpo estranho esofágico radiopaco em cão: Relato de caso
}

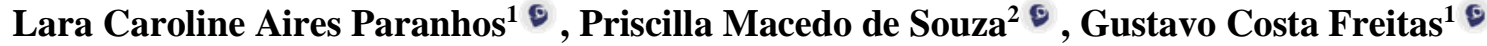 \\ ${ }^{1}$ Médico(a) Veterinária inscrito(a) no Programa Profissional de Aprimoramento em Medicina Veterinária da Universidade Federal do \\ Tocantins, Departamento de Clínica Médica de Pequenos Animais, Araguaína, Tocantins, Brasil. \\ ${ }^{2}$ Professora da Universidade Federal do Tocantins, Departamento de Diagnóstico por Imagem, Araguaína, Tocantins, Brasil. \\ *Autor para correspondência, E-mail: lara_carolline@hotmail.com
}

\begin{abstract}
Resumo. Este trabalho teve como objetivo relatar o caso de um cão com corpo estranho esofágico, apontando os sinais radiográficos em decorrência deste, além de dissertar sobre os tipos, radiopacidade, localização frequente e conduta do médico veterinário radiologista diante desta situação. Casos de corpo estranho é relativamente comum em cães. Pode ter importância clínica ou não, é recomendado a realização de exames radiográficos simples ou contrastados que pode ser complementado com a ultrassonografia e outras modalidades como a tomografia computadorizada, ressonância magnética e endoscopia. O diagnóstico foi baseado na anamnese, sinais clínicos e imagens radiográficas do corpo estranho. Posteriormente, o paciente foi encaminhado ao centro cirúrgico para realização da esofagostomia e toracotomia para remoção dos fragmentos ósseos (corpo estranho). A utilização do exame radiográfico foi de extrema importância para determinar o diagnóstico de obstrução esofágica, possibilitando agilidade no encaminhamento e na sua intervenção.
\end{abstract}

Palavras-chave: afecção digestiva superior, obstrução, radiologia

\section{Radiographic signs of radiopaque esophageal foreign body in a dog: Case report}

\begin{abstract}
The aim of this study is to report the case of a dog with an esophageal foreign body in a dog, point out the radiographic signs due to that. In addition to talking about the types, radiopacity, frequent location and conduct of the veterinary radiologist in this situation. Foreign body cases are relatively common in dogs. It may be or not a clinical relevance, and it is recommended to be performed with contrasted or simple radiographic exams, complemented with ultrasound and other modalities such as computed tomography and endoscopy. The diagnosis was based on anamnesis, clinical signs and radiographic images of the foreign body. Subsequently, the patient was referred to the operation room for esophagotomy and thoracotomy to remove bone fragments (foreign bodies). The use of radiographic examination was extremely important to determine the diagnosis of esophageal obstruction, allowing for quick referral for surgical intervention.
\end{abstract}

Keywords: Obstruction, radiology, upper digestive disorder

\section{Introdução}

Entende-se por corpo estranho qualquer material ingerido pelo animal e que não possa ser digerido, podendo em alguns casos causar obstrução intraluminal total ou parcial (Camelo Júnior et al., 2019). Os cães costumam ingerir corpos estranhos de forma indiscriminada, como brinquedos de plásticos, sacos de cozinha, pedras entre outros objetos. Já os felinos ingerem com maior frequência materiais lineares e entre eles destacam-se fios de tecido, pedaços de barbante, linha de costura, pano ou fio dental (Parra et al., 2012). 
Os corpos estranhos ingeridos são encontrados com maior frequência na entrada torácica, na base cardíaca e cranial ao hiato esofágico devido ao estreitamento do esôfago. Quando o corpo estranho se aloja no interior do esôfago, há um aumento do peristaltismo. Caso o corpo estranho permaneça nessa região por alguns dias, poderá provocar ondas peristálticas repetidas que podem causar necrose na parede esofágica. Como consequência, se estabelece um quadro de esofagite, interferindo na sua motilidade e na pressão do esfíncter esofágico caudal, causando alteração na função neuromuscular e reduzindo o peristaltismo. Além disso, a lesão na mucosa esofágica pode contribuir para o desenvolvimento de pneumonia por aspiração (Rodrigues et al., 2016).

Em obstruções completas, quando não há passagem natural do objeto pelo trato gastrointestinal, podem ocorrer complicações mais graves como choque hipovolêmico ou peritonite séptica e, nessas condições, a estabilização rápida e a intervenção cirúrgica são necessárias. A técnica cirúrgica é decidida de acordo com a causa e a localização da obstrução (Oliveira \& Martins, 2017).

Relatos de corpo estranho no trato gastrointestinal é frequente em animais domésticos. Utilizam-se exames radiográficos simples ou contrastados, complementando com a ultrassonografia e outras modalidades como a tomografia computadorizada, endoscopia óptica e ressonância magnética (Silva et al., 2016). No que se refere ao diagnóstico por imagem, as radiografias com ou sem contraste e a endoscopia são os métodos mais acessíveis. Os corpos estranhos podem ser visualizados em radiografias simples quando são mineralizados ou metálicos, como osso, projétil balístico, agulhas e anzol (Parra et al., 2012).

Muitas doenças esofágicas podem ser achadas por radiografia simples, já que a mistura de ar, líquidos e alimentos no esôfago podem delineá-lo, e assim, preenchê-lo parcialmente. As radiografias simples podem auxiliar na constatação de alterações estruturais tais como dilatação segmentar ou generalizada do esôfago, conteúdo anormal no esôfago devido à ausência de peristaltismo normal e presença de pneumonia aspirativa. Em caso de animais com histórico de regurgitação, a ausência de alterações radiográficas não exclui a possibilidade de doença esofágica, sendo necessário a realização do esofagograma para melhor elucidação do diagnóstico (Thrall, 2013). Alterações peristálticas, hipomotilidades, compressões ou corpos estranhos radiolucentes causando obstrução podem passar despercebidos nas avaliações radiográficas simples. Um esofagograma contrastado pode auxiliar na diferenciação entre estas condições. É contra indicado para a ingestão de bário as evidências radiográficas de pneumotórax, pneumomediastino e líquidos pleurais vistas na radiográfica simples, os quais são sinais de uma possível perfuração esofágica, iodo orgânico hidrossolúvel é o mais indicado nesses casos. $\mathrm{O}$ uso de sulfato de bário em casos de ruptura esofágica pode trazer consequências graves ao paciente, este livre na cavidade torácica se adere na parede provocando uma intensa reação inflamatória do tipo granulomatosa (Assunção, 2017). A radiografia é o método mais disponível e ainda muito úteis no caso de corpos estranhos esofágicos (Moraillon et al., 2013). É indicado radiografar antes da cirurgia, pois, o objeto pode sair do esôfago e se localizar no estômago, intestino delgado ou no cólon. Os corpos estranhos no cólon geralmente são eliminados com facilidade, menos os com bordas pontiagudas que podem se prender ao ânus (Camelo Júnior et al., 2019).

Quando o corpo estranho é pequeno, não é tóxico e radiopaco, é recomendado o acompanhamento do animal por um período de 7 a 10 dias pela radiografia para monitorar a localização do corpo estranho. Recomenda-se a remoção imediata caso se desenvolvam sinais clínicos de obstrução esofágica como disfagia, regurgitação e sialorreia. O tamanho, conteúdo e grau de acometimento do corpo estranho no animal determina seu prognóstico (Birchard \& Sherding, 2008). Na avaliação radiográfica do esôfago devemos incluir a partir da base da língua até o diafragma. No posicionamento ventrodorsal a coluna vertebral fica sobreposta ao esôfago é necessário que se realize uma radiografia látero-lateral. A incidência ventrodorsal o cão é posicionado em decúbito dorsal, com os membros anteriores esticados, o esterno deve ficar sobreposto a coluna vertebral. Na látero-lateral, o cão é colocado em decúbito lateral com as patas anteriores sentido cranialmente (Anjos et al., 2019). A endoscopia e a radiografia são os exames de escolha em caso de corpo estranho gástrico ou esofágico. A endoscopia permite o diagnóstico e, em certos casos, retirar o corpo estranho (Moraillon et al., 2013).

\section{Relato de caso}

Foi atendido na Clínica Veterinária Universitária - EMVZ - UFT em maio de 2019 um cão, fêmea, sem raça definida, 2 anos e 7,5 $\mathrm{kg}$ tendo como queixa principal, informada pela tutora, dificuldades 
respiratórias (dispneia), sialorreia e dor na região torácica. Relatou ainda a possibilidade da paciente ter ingerido pedaços de ossos de galinha no mesmo dia pela manhã. Durante o exame físico, foi contatado mucosas normocoradas, tempo de preenchimento capilar de 2 segundos, frequência cardíaca 120 batimentos por minuto, frequência respiratória 20 movimentos por minuto, normohidratada, pulso forte e rítmico estado geral apático. De acordo com as informações obtidas durante a anamnese e pelo exame físico, a principal suspeita clínica era de ingestão de corpo estranho.

Posteriormente, a paciente foi encaminhada para o setor de diagnóstico por imagem, onde foi realizado o exame radiográfico simples do tórax, nas projeções lateral e ventrodorsal. Foram observados então as seguintes alterações: deslocamento ventral de traqueia torácica, dilatação segmentar de esôfago torácico cranial e médio, cavidade gástrica distendida e preenchida por conteúdo homogêneo (gasoso), três corpos estranhos radiopacos no esôfago torácico em região $4^{\circ}$ a $6^{\circ}$ costela próximos a base do coração, medindo $2,2 \mathrm{~cm}$ x $0,8 \mathrm{~cm}$ e $0,9 \mathrm{~cm}$ x $0,8 \mathrm{~cm}$ e $0,7 \mathrm{~cm}$ x $0,5 \mathrm{~cm}$, com característica de estrutura mineralizada semelhante a osso.
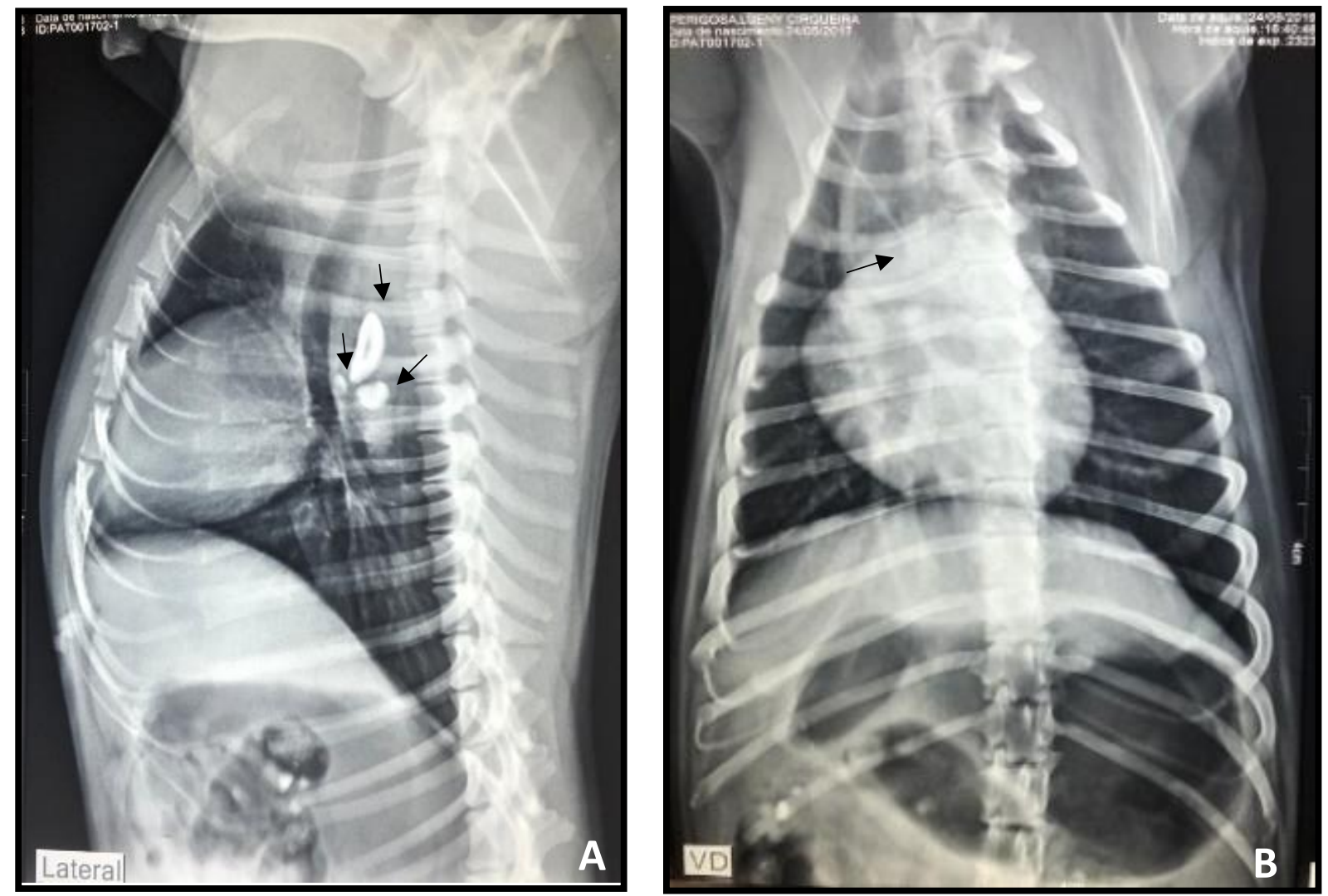

Figura 1. (A) - Projeção látero-lateral direita. Podemos identificar três estruturas radiopacas localizadas em porção medial de esôfago torácico (indicados nas setas pretas). (B) - Projeção ventrodorsal. A análise radiográfica na projeção ventrodorsal é possível notar a sobreposição do coração com o material que é mais radiopaco que o órgão, entre a $4^{\circ}$ e $6^{\circ}$ costela (indicado na seta preta). Fonte: Arquivo CVU - UFT 2019

A paciente foi encaminhada para o centro cirúrgico para realizar uma toracotomia e uma esofagostomia. Foram retirados três corpos estranhos com estruturas condizentes com fragmentos ósseos. $\mathrm{O}$ animal foi encaminhado para internação em uma clínica particular. Quinze dias após o paciente retornou a clínica para retirada dos pontos mostrando completa melhora e o local da cirurgia cicatrizado.

\section{Discussão}

$\mathrm{Na}$ obstrução esofágica por corpos estranhos intraluminais os sinais clínicos são diversificados e dependem da duração, localização e do tipo da obstrução. Entre eles, pode-se incluir náusea, sialorreia, mímica de vômito, inapetência, inquietação, depressão, desidratação e angústia respiratória (Camelo Júnior et al., 2019). A paciente em questão apresentava como sinais clínicos apatia, hiperalgia na região mediastínica média e sialorreia, que corroboram com a literatura. 
A comorbidade pode ainda ser classificada de duas maneiras, em fase aguda ou crônica. Animais que cursam com a fase aguda normalmente tendem a apresentar engasgos ou regurgitação logo após refeição, já pacientes com obstrução de caráter crônico, pode ser acrescido aos sinais a perda de peso (Birchard \& Sherding, 2008). O paciente em questão apresentava a forma aguda da doença uma vez que este foi diagnosticado logo após o aparecimento dos primeiros sinais clínicos.

Como método de diagnóstico, foi realizada a radiografia simples que é comumente utilizada na rotina de clínicas e hospitais veterinários. Objetos radiopacos, tais como metal ou osso, são de fácil reconhecimento quando equiparados com corpos estranhos não radiopacos tais como plástico e cartilagem, os quais aparecem como uma opacidade focal de tecidos moles na região do esôfago. No caso de corpo estanho não radiopaco, radiograficamente pode se assemelhar a uma neoplasia esofágica, um abscesso esofágico, formação mediastínica, hérnia para esofágica ou formação pulmonar (Thrall, 2013). Pelas imagens radiográficas observamos estruturas de rádio opacidade compatíveis com material mineralizado como osso, de acordo com a escala de rádio densidade, esse tipo de material é facilmente reconhecível quando comparados com materiais não radiopacos. No exame radiográfico foi identificado três estruturas radiopacas em esôfago torácico.

Em casos de obstrução esofágica geralmente observamos sinais radiográficos de dilatação esofágica, essa pode ser segmentar ou generalizada, dependendo da causa e da localização da doença, podendo ser também funcional ou mecânica. A dilatação generalizada é tipicamente causada por doença funcional e a dilatação segmentar geralmente é causada por corpo estranho (Silva et al., 2016). Como observado nas imagens radiográficas do animal do relato, havia um aumento da radio opacidade água periadjacente e cranial aos corpos estranhos possibilitando a visualização da dilatação segmentar do esôfago cranial e medial, não é possível visualizar o esôfago caudal, a não visualização e normal em radiografias simples.

Geralmente os corpos estranhos são encontrados no trecho cranial da porção torácica, na base do coração e no hiato esofágico (Camelo Júnior et al., 2019). Como observado neste caso o material radiopaco posicionado na base do coração, esse é um local comum de alojamento de corpos estranhos esofágicos devido à presença de elementos anatômicos extra esofágicos que limitam sua dilatação neste local.

A maior parte dos corpos estranhos radiopacos possuem sinais radiográficos que são melhor identificados pelas radiografias simples de boa qualidade, caso se suspeita de perfuração esofágica, são recomendados materiais de contraste iodados orgânicos e hidrossolúveis ou iohexol (Assunção, 2017). Não foi possível a realização do exame contrastado devido o animal se encontrar bastante apático e com dor e também pela rádio opacidade do material que possibilitou a visualização na radiografia simples.

A endoscopia é indicada se radiografias simples ou contrastada revelarem massa ou corpo estranho, obstrução esofágica, tumor, hérnia hiatal ou inflamação são suspeitos (Nelson \& Couto, 2015). A endoscopia não foi realizada pois a clínica não possuía o aparelho, e também por não saber se havia ruptura no esôfago, então o método não era indicado.

É muito importante obter a radiografia imediatamente antes da cirurgia para garantir que o corpo estranho não se moveu e que não há nenhuma evidência de perfuração (Camelo Júnior et al., 2019). As radiografias realizadas no referido caso foram feitas previamente antes da cirurgia, o animal foi atendido em caráter de emergência e logo encaminhado para o centro cirúrgico a qual foi realizada com sucesso.

\section{Conclusão}

A realização do exame radiográfico em casos de corpos estranho esofágico e de extrema importância dentre outros exames, pois proporciona ao médico veterinário um maior conhecimento sobre a localização, aspecto e tamanho do objeto além de ser uma técnica mais simples e de fácil detecção. A radiografia simples e a contrastada são de grande valia pois é rápida e traz informações importantes ao prognóstico do animal e ao cirurgião.

\section{Referências}

Anjos, Á. M., Ribeiro, J. P. G., \& Aires, D. M. P. (2019). Importância do exame radiográfico no diagnóstico de megaesôfago em cães: Revisão de literatura. Revista Eletrônica da Faculdade de Ceres, 8(1), 11. DOI: https://doi.org/10.36607/refacer.v8i1.4485 
Assunção, G. A. (2017). Corpos estranhos esofágicos em cães e gatos: revisão de literatura. Universidade Federal do Rio Grande do Sul.

Birchard, S. J., \& Sherding, R. G. (2008). Manual Saunders: clínica de pequenos animais. In Ed. Roca (Vol. 3).

Camelo Júnior, F. A. A., Franco, T., Feijó, V. M., Brito, R. S. A., Medeiros, H. Z., Fonseca, M. G. M., Madruga, P. A., Priario, D. F., Jorge, S., \& Squeff Filho, J. (2019). Esofagotomia torácica para remoção de corpo estranho em um cão: Relato de caso. PUBVET, 13(7), 1-5. DOI: https://doi.org/10.31533/pubvet.v13n7a380.1-5

Moraillon, R., Legeay, Y., Boussarie, D., \& Sénécat, O. (2013). Manual Elsevier de Veterinária: Diagnóstico e tratamento de Cães, gatos e animais exóticos (7th ed.). Elsevier.

Nelson, R. W., \& Couto, C. G. (2015). Medicina interna de pequenos animais (Issue 1). Elsevier Editora.

Oliveira, R. C. B., \& Martins, N. C. (2017). Corpo estranho obstrutivo intestinal em cão: Relato de caso. Simpósio de Trabalho de Conclusão de Curso e Seminário de Iniciação Científica, 2258-2263.

Parra, T. C., Berno, M. D. B., Guimarães, A., Andrade, L. C. A., Mosquini, A. F., \& Montanha, F. P. (2012). Ingestão de corpo estranho em cães-Relato de caso. Revista Científica Eletrônica de Medicina Veterinária, 18.

Rodrigues, D. S. A., Soares, L. L. S., Rodrigues, R. P. S., Santos, M. M., Barros, D. A., Barbosa, Y. G. S., \& Rodrigues, M. C. (2016). Esofagotomia torácica para remoção de corpo estranho associado a megaesôfago em cão. PUBVET, 10(8), 615-618. DOI: https://doi.org/10.22256/pubvet.v10n8.615$\underline{618}$

Silva, F. F. S., Ré, B. G., Pinto, A. C. B. C. F., Lorigados, C. A. B., Unruh, S. M., \& Kanayama, L. M. (2016). Diagnóstico por imagem de corpo estranho gastrointestinal em cães e gatos: estudo retrospectivo de 157 casos. Revista de Educação Continuada Em Medicina Veterinária e Zootecnia Do $C R M V-S P, 14(3), 54-55$.

Thrall, D. E. (2013). Textbook of veterinary diagnostic radiology. Elsevier Health Sciences.

\section{Histórico do artigo:}

Recebido: 21 de outubro de 2020. Aprovado: 8 de dezembro de 2020

Disponível online: 5 de março de 2021.
Licenciamento: Este artigo é publicado na modalidade Acesso Aberto sob a licença Creative Commons Atribuição 4.0 (CC-BY 4.0), a qual permite uso irrestrito, distribuição, reprodução em qualquer meio, desde que o autor e a fonte sejam devidamente citados. 